\title{
COLONIZACIÓN DE ÁREAS DEFORESTADAS DE LA AMAZONIA PERUANA POR LA LECHUZA DE LOS ARENALES Athene cunicularia (MOLINA, 1782)
}

\section{COLONIZATION OF DEFORESTED AREAS IN THE PERUVIAN AMAZON BY THE BURROWING OWL Athene cunicularia (MOLINA, 1782)}

\author{
Víctor Pulido ${ }^{1}$
}

\begin{abstract}
Resumen
La distribución bien conocida de la lechuza de los arenales (Athene cunicularia) en el Perú abarca los bosques secos del noroeste, el desierto costero, las vertientes occidentales de los Andes y los valles interandinos. Sin embargo, en los últimos 20 años se le ha visto en la Amazonia peruana, en las regiones de Madre de Dios, Loreto y Ucayali. En el país se registran cuatro subespecies de A. cunicularia, pero, hasta ahora no se ha podido definir con precisión la o las subespecies que han ampliado su rango de distribución en la Amazonia peruana. A. cunicularia puede ser considerada una especie indicadora de hábitats antropogénicos, en donde se ha sustituido el bosque primario por actividades agropecuarias o por bosques abiertos muy degradados. En el presente trabajo se efectúa una revisión bibliográfica y el seguimiento de los avistajes de A. cunicularia en las zonas boscosas de la Amazonia peruana en proceso de degradación con el objetivo de evaluar la expansión en el rango de distribución de la especie en Perú. Se encontraron 80 registros nuevos y de nidificación de la especie en sitios donde no se habían reportado previamente reproduciéndose. Se confirma la ampliación del rango de distribución de la especie en la colonización de la Amazonia, desde el lado peruano, siguiendo la ruta de los ríos que desciende por los valles interandinos, atraviesa la selva alta y llegan a la selva baja, en el avance del establecimiento sobre terrenos de cultivo y asentamientos humanos.
\end{abstract}

Palabras clave: Athene cunicularia, distribución geográfica, Amazonia, actividades antropogénicas.

\begin{abstract}
The well-known distribution of the burrowing owl (Athene cunicularia) in Peru encompasses the dry forests of the northwest, the coastal desert, the western slopes of the Andes, and the interAndean valleys. However, in the past 20 years, this species has been seen in the Peruvian Amazon, in the regions of Madre de Dios, Loreto, and Ucayali. Although four subspecies of A. cunicularia are registered in the country, it has not yet been possible to precisely define the subspecies that have expanded their distribution range in the Peruvian Amazon. A. cunicularia can be considered an indicator species of anthropogenic habitats, where the primary forest has been replaced by agricultural activities or highly degraded open forests. In the present work, a bibliographic review and monitoring of A. cunicularia sightings in forested areas of the Peruvian Amazon in the degradation process is carried out with the objective of evaluating the expansion in the distribution range of the species in Peru. Eighty new and nesting records of the species were found in places where it had not been reported previously breeding. Broadening of the distribution range of this species in the Amazon colonization is confirmed, from the Peruvian side, following the route of the rivers that descends through the inter-Andean valleys, crosses the high jungle, and reaches the low jungle, in the advance of the establishment on farmland and human settlements.
\end{abstract}

Key words: Athene cunicularia, geographical distribution, Amazonia, anthropogenic activities.

\section{Introducción}

El Perú es el segundo país en extensión forestal en América Latina, con 72 millones de hectáreas de bosques y el cuarto con mayor área de bosques tropicales húmedos (MINAM, 2009). Como ocurre en otros países tropicales, sus bosques están sometidos a una extensa deforestación con fines agropecuarios y mineros $\mathrm{y}$, asimismo, sufren severa degradación, especialmente como consecuencia de la explotación forestal, la caza y la contaminación minera. Se estima conservadoramente que en el Perú se han destruido más de 11 millones de hectáreas de bosques y que no menos del $50 \%$ de los que aún están en pie han sido degradados (Dourojeanni, 2019b).

La deforestación y la pérdida de fuentes de agua, especialmente en las cabeceras de cuencas, asociadas al impacto del cambio climático, tienen un rol determinante en el proceso de aridización y sabanización de la Amazonia, por lo que los cambios ambientales vienen ocasionando la colonización por parte de especies que responden a hábitats más secos (Dourojeanni, 2019a; Rojas et al., 2019). La deforestación está concentrada en las regiones de San Martín (19.42\%), seguida de Loreto (14.68\%) y 
Amazonas (12.30\%) (MINAM, 2015). En cambio, la degradación forestal es muy generalizada, afectando todas las regiones, especialmente la de Pucallpa (MINAM, 2013).

La acción acumulada de la deforestación, fragmentación y degradación de hábitats, así como la generación de hábitats sucesionales, están causando cambios en la composición de la biodiversidad en la medida que muchas especies, propias de este ecosistema, están desapareciendo, y que, simultáneamente, otras especies están colonizando estos ambientes (Harvey et al., 2014). Esa pérdida y fragmentación de los hábitats naturales disminuye las poblaciones de las especies y es una de las causas de la desaparición de otras (Granizo et al., 2002). En este escenario, la sucesión se ve influenciada por eventos probabilísticos, por la biología de las especies, por su forma de interactuar con plantas y animales, el suelo y el clima del lugar (Gaston, 2005; Stouffer, 2020). Estos factores dan origen a una determinada composición y, además, influyen en la velocidad a la que un bosque recupera su estructura y funcionamiento original (Sekercioglu et al., 2008). Los estudios sobre el estado de un hábitat sirven de base para señalar prioridades para la conservación de la diversidad biológica y el medio ambiente global (Núñez, 2008).

La biodiversidad amazónica está conformada por millones de especies, entre las cuales las aves constituyen uno de los taxones más conocidos que han sido identificados como indicadores del cambio climático y cuyas poblaciones están siendo afectadas en los bosques tropicales de tierras bajas debido a que algunas especies de árboles proveedores de frutas e insectos se han dispersado hacía hábitats de mayor elevación, donde encuentran microclimas más adecuados para su supervivencia (Mittermeier et al., 2003; Colwell et al., 2008; Locatelli et al., 2009; De Sy et al., 2015). La gran cantidad de datos recolectados, proporcionados por cientos de miles de observadores de aves, ha permitido que se tenga información cada vez más precisa y actualizada acerca de los efectos de la destrucción de los bosques y el cambio climático sobre las poblaciones de aves que habitan en los ecosistemas de los bosques de América Latina, donde se concentra parte de la mayor diversidad de especies del mundo (Sekercioglu et al., 2011).

El conocimiento sobre la relación entre las aves y los factores ambientales permite entender el efecto de los impactos humanos sobre la diversidad de la avifauna. Las aves responden a una serie de condiciones, como la variación de la densidad poblacional, y las respuestas a los diferentes impactos están determinadas por características particulares como el tamaño de los ejemplares, hábitos alimenticios, selección de sitios de nidificación, capacidad de dispersión, etc. De allí que las especies puedan presentar diferentes tipos de respuestas: disminución o aumento de su abundancia, cambios en su distribución, desplazamiento poblacional a lugares con alteraciones ambientales e, incluso, su desaparición local (Lepczyk et al., 2008).

La presencia de las aves contribuye al análisis de los procesos ecológicos complejos como gradientes en la cobertura del dosel de bosques, procesos de fragmentación y urbanización (Stouffer, 2020). Las aves son consideradas como buenos indicadores de los cambios ambientales debido a que vuelan a grandes distancias, están ampliamente distribuidas y son sensibles a cambios en el ecosistema, como la contaminación ambiental, alteración del hábitat y el efecto de las actividades humanas (Lara, 2020). En general, responden a la composición y estructura de la vegetación, por lo que son utilizadas como indicadores de impacto ambiental en los ecosistemas boscosos, revelan el grado de la destrucción o la modificación del hábitat sobre otras especies y grupos, y cumplen un importante rol en el mantenimiento de funciones ecológicas claves dentro de los sistemas forestales, tales como la diseminación de semillas y la polinización (WWF, 2004; Salas \& Mancera, 2020). Este aspecto cobra especial relevancia si se tiene en cuenta que cerca al $50 \%$ de los árboles en los bosques húmedos tropicales tienen semillas que son dispersadas especialmente por las aves, que también cumplen una serie de funciones ecológicas claves para el mantenimiento de la integridad de los ecosistemas forestales (Canterbury et al., 2000).

En la Amazonía se registran más 1300 especies de aves, de las que 250 son endémicas. No obstante, familias importantes como Columbidae y Strigidae no incluyen especies endémicas en la Amazonia (Nores, 2000). De un total de 73 especies de aves rapaces en el Perú (Cathartidae 6, Pandionidae 1, Accipitridae 18, Tytonidae 1, Strigidae 29, Falconidae 18), cerca de 30 viven en las tierras bajas del bosque amazónico (Schulenberg et al., 2010; SERFOR, 2018; Plenge, 2021). Sin embargo, muchas de ellas son extremadamente cautelosas, difíciles de ver y ocurren en densidades muy bajas.

La lechuza de los arenales, Athene cunicularia, es un ave de presa terrestre e inconfundible cuyo plumaje se asemeja al color del suelo del hábitat donde reside, de actividad diurna y crepuscular, de dieta generalista y oportunista; se alimenta de pequeños roedores, aves, reptiles, anfibios, artrópodos y gasterópodos (Medina et al., 2013; Mamédio dos Santos et al., 2017). Es sedentaria y tolera niveles considerables de alteración antropogénica en sus hábitats, lo cual es una ventaja porque desarrolla una amplia capacidad para adaptarse a la disponibilidad de presas según su abundancia o escasez, y que influye en su versatilidad para adaptarse a nuevas condiciones y resistir cambios drásticos de hábitat (Wade \& Belthoff, 2016; Lincer et al., 2018). Se refugia en madrigueras excavadas en el suelo por otras especies animales, o en termiteros, las cuales son 
utilizados para la cría y anidamiento (Gomes et al., 2013).

A. cunicularia es de amplia distribución, abarca desde Canadá hasta Tierra del Fuego, en el extremo sur del Continente Americano (Macías \& Conway, 2015), y ocupa diferentes hábitats desde el nivel del mar hasta los 4000 metros de elevación. Comprende 20 subespecies, de las cuales cuatro se han registrado en Perú: A. c. punensis, del suroeste de Ecuador al sur en la costa al noroeste de Perú; $A$. $c$. nanodes en la costa del suroeste de Perú al norte de Chile; A. c. juninensis en los Andes de Perú, oeste de Bolivia y noroeste de Argentina; y A. c. boliviana que se distribuye en la Amazonia de Bolivia, en los departamentos de Beni y Santa Cruz y, probablemente, se encuentra también en Madre de Dios, Perú (Lincer, et al., 2018). Los estudios de comportamiento con A. cunicularia permiten comprender cómo sus poblaciones enfrentan el cambio antrópico, principalmente aquel relacionado al avance urbano (Carrete \& Tella, 2017; Rebolo-Ifrán et al., 2017). La colonización de entornos humanizados por esta especie se ha relacionado con la variabilidad interindividual en su miedo a los humanos y se ha comprobado que este comportamiento es hereditario y constante a lo largo de la vida adulta de A. cunicularia. (Carrete \& Tella, 2017). Así mismo, utiliza para nidificar, en todo su rango de distribución, áreas abiertas sin árboles cercanos, típicamente zonas donde existen herbívoros que mantienen el pasto corto y mamíferos excavadores que, además de pastar, construyen madrigueras que luego las lechuzas utilizan para ellas (Borsellino, 2017; Rocha et al., 2021).

Hasta el siglo pasado, A. cunicularia no había sido registrada en la Amazonía peruana, sin embargo, en los últimos veinte años se ha reportado la ocurrencia de la especie en estos hábitats. En este sentido, es de particular importancia evaluar la posible modificación del rango de distribución de esta especie en el Perú. El objetivo de la presente investigación es efectuar una revisión bibliográfica y el seguimiento de los avistajes de A. cunicularia a fin de verificar la expansión de esta especie en las zonas boscosas de la Amazonia peruana en proceso de degradación.

\section{Métodos}

Desde 1977, se han efectuado evaluaciones en lugares de la selva alta y baja en las Regiones de Madre de Dios, Pucallpa, San Martín, Loreto, Amazonas, Cajamarca y Cusco que incluye la observación directa con binoculares sobre el hábitat, dieta alimenticia, comportamiento, desplazamientos altitudinales, distribución y estado de conservación de A. cunicularia (Pulido \& Aguilar, 1979; Pulido, 1982; Pulido et al., 2007; Pulido et al., 2013). Además, se ha consultado la base eBird (TheCornellLab of Ornithology, 2020) en donde se ha registrado a $A$. cunicularia en diversos lugares de la Amazonia, así como se ha complementado dicha información a través de consultas por correo electrónico a investigadores que trabajan en aves en la Amazonía.

Se realizó una búsqueda sistemática utilizando Scopus, Web of Science, Scielo y Google Académico, en español e inglés, haciendo uso de las palabras clave: "A. cunicularia", "lechuza de los arenales", "distribución geográfica", "Amazonia”, "actividades antropogénicas", "Strigidae", "egagrópilas" y "regúrgitos". Se tuvieron en cuenta 63 artículos científicos relevantes con datos sobre poblaciones en Perú o países aledaños, publicados entre 1973 y 2021. Datos de distribución de la especie fueron obtenidos de la base de datos de GBIF (2020), que incluyen los registros de múltiples bases de datos, tanto de colecciones científicas como de observaciones de campo (i.e. eBird); y también, de la información bibliográfica revisada. Adicionalmente, se utilizó como base el mapa de distribución a nivel mundial de BirdLife International (2016) y a nivel nacional el de Schulenberg et al. (2006).

\section{Resultados y discusión \\ Athene cunicularia en Brasil y Bolivia}

En Brasil la especie se encuentra en campos abiertos, pastizales, bancos de arena, sabanas y gramadales, campos cultivados y ganaderos, y en áreas urbanas es común en ciudades, parques urbanos y canchas de golf; en los bosques de arena blanca (Alonso et al., 2013).

En la Amazonía brasileña se ha registrado en sabanas naturales o en espacios abiertos. El primer registro fue en 1966 en el Estado de Roraima (Gomes et al., 2013); luego, se reportó en 1991, en Alta Floresta, norte de Mato Grosso (Zimmer et al., 1997); en 1992 se recolectó una muestra en Pará (Aleixo \& Poletto 2007) y, en ese mismo año, se registró en zonas de pastizales en el Estado de Amazonas, (Cohn et al., 1997); en 1994 se tuvo un nuevo registro en Alta Floresta (Zimmer et al., 1997). En Manaos, el primer registro fue en 1992 por Bierregaard, quien observó a un solo individuo en un área de pastos y en julio de 1994, se registró un individuo en la misma ubicación (Cohn et al., 1997). Entre el 2006 y 2012, fueron reportados, algunos ejemplares en los municipios de Presidente Figueiredo; también ha sido registrada en los bosques de arena blanca, pasturas y áreas modificadas por el hombre en el noroeste de Rondônia, en el sur de la Amazonia de Brasil (Guilherme et al., 2018).

En la Amazonia de Bolivia, el 20 de febrero de 2007, se registraron tres individuos de A cunicularia en el trayecto de la carretera Cobija - Puerto Rico, cerca del poblado del Porvenir, Provincia Nicolás Suárez, Departamento de Pando, en un pastizal de uso agropecuario, que anteriormente fue un bosque y que ha podido favorecer a esta especie para colonizar nuevas áreas (Miserendino, 2007). El segundo registro para la Amazonía boliviana fue el 16 de junio de 2015 
Julio - Diciembre 2021

en la "Carretera 19 Extrema", en un área deforestada de la localidad de Mukden, donde se reportaron dos ejemplares, a una elevación de $120 \mathrm{~m}$, en el Departamento de Pando (Richard et al., 2017) Athene cunicularia en el Perú

En el Perú, ocupa una amplia variedad de hábitats en zonas áridas, bosques de algarrobo, chaparral, campos abiertos, lomas costeras, planicies desérticas, dunas, desiertos con cactáceas o tillandsiales en suelos arenosos de los ríos, humedales, terrenos de cultivo, quebradas, monte ribereño, sabanas, pajonal de puna y estepas; en la vertiente occidental y en las zonas andinas prefiere lugares rocosos (Salinas et al., 2006; Pulido et al., 2013).

La especie ha sido registrada en las vertientes occidentales de los Andes, los valles interandinos y la puna (Schulenberg et al., 2006). Anteriormente desconocida en la Amazonía peruana, esta especie se ha encontrado en pequeñas cantidades en áreas agrícolas y a lo largo de ríos en las Regiones de Loreto, Ucayali y Madre de Dios (Figura 1). Aunque los registros sobre A cunicularia son escasos, se tienen algunas evidencias de que se reproduce en la Amazonia baja del Perú desde los últimos 20 años. El primer registro reportado fue en el Parque Nacional Manu, Región Madre de Dios (Yábar, 2008). En los alrededores de Puerto Maldonado, A. cunicularia fue registrada en los pastizales de Triunfo, Albergue y Palma 1 (Los Ángeles), lo que constituye el primer reporte de esta especie en los alrededores de Puerto Maldonado (Butrón \& Tapia, 2010), ampliando así su área de distribución establecida por Schulenberg et al. (2006).

Posteriormente, Harvey et al. (2014) han observado en tres ocasiones, en campos alrededor del pueblo de Atalaya y en las islas fluviales del río Tambo y Río Ucayali: dos adultos y dos jóvenes apenas capaces de volar, el 17 octubre de 2008 en un campo al sureste de Atalaya; dos adultos, incluida una hembra con un huevo calcificado en el oviducto, y que fueron colectados en una isla en el río Tambo el 24 de julio de 2010. Estos registros constituyen para la Región de Ucayali las primeras observaciones documentadas acerca de su reproducción en la Amazonía peruana.

Delzo et al. (2019) la registra para el Distrito de Calleria, Provincia de Coronel Portillo en Pucallpa, Región Ucayali. Adicionalmente, el 26 de febrero de 2020 se registraron dos individuos adultos de A. cunicularia en un canal de drenaje del Aeropuerto Internacional Capitán FAP David Abensur Rengifo, que se encuentra ubicado en el Departamento de Ucayali, Provincia de Coronel Portillo, en la ciudad de Pucallpa, cuyas coordenadas geográficas son: latitud: $8^{\circ}$ 19' 59" S, longitud 74 37' 59" W, elevación de 154 msnm. Aunque no se encuentran documentadas, observadores experimentados de aves la registran para las Regiones de San Martín, Amazonas, Cajamarca, Huánuco, Pasco, Junín, Huancavelica, Ayacucho,
Apurímac, Cusco, en chacras, zonas de purmas y jardines de alojamientos (Tabla 1).

Un aspecto de particular interés es la sucesión de hábitats en el valle superior de Ucayali alrededor del pueblo de Atalaya, en las islas fluviales del río Tambo y Río Ucayali que han sido fuertemente influenciados por la actividad humana. Las áreas abiertas han aumentado como resultado de la colonización y la expansión humana, y esta tendencia estaría impulsando la aparición de una serie de especies de aves que se han considerado raras o locales en la cuenca del Amazonas (Harvey et al., 2014). A. cunicularia sería un colono reciente de la Amazonía peruana, y aunque las actividades antrópicas serían perjudiciales para las especies más especialistas, también podrían aportar beneficios a muchas otras especies más generalistas como lo es A. cunicularia, aprovechando el cambio en la estructura ambiental y aumentando entonces su área de distribución (Pulido et al., 2021).

Los hábitats sucesionales y periféricos podrían ser importantes no solo para los residentes, sino también por la diversidad de los migrantes boreales y australes, y parece probable que estas especies, así como las del interior del bosque, se vean influenciadas a medida que continúa la deforestación. Esa es la situación del alitorcido rufo Cnipodectes superrufus de la familia Tyrannidae, que habita en el suroeste de la Amazonia, en Ucayali, Cuzco y Madre de Dios, en el sur de Perú (Fjeldså, 2020), que tiene una baja capacidad de dispersión y no logra migrar hacia nuevos hábitats. Muchas de las especies registradas en este hábitat están muy extendidas en áreas abiertas en la cuenca del Amazonas, y otras son raras y locales en la Amazonía, incluidas A. cunicularia y Gampsonyx swainsonii (Harvey et al., 2014).

Origen de la población de A. cunicularia en la Amazonía del Perú

El Comité de Registros de Aves Peruanas (CRAP, 2015) considera que las poblaciones amazónicas peruanas de $A$. cunicularia no pertenecen a ninguno de los taxones de los Andes o de la costa del Pacífico, como es el caso de A. c.punensis, A. c.nanodes y A. c. juninensis, y que los taxones presentes en las tierras bajas de la Amazonia peruana tampoco corresponden a A.c. boliviana. El CRAP (2015) recomienda una reevaluación actual de las taxa que componen A. cunicularia para establecer las poblaciones que podrían haber ampliado su rango de distribución hacia la Amazonía del Perú. Mientras no se tenga mayor información, existiría la incertidumbre de si es una o son más las subespecies que actualmente han ampliado su rango distribución en la Amazonia peruana.

Guilherme et al. (2018) sugiere que en Brasil la expansión de la distribución de la especie no está ocurriendo linealmente, sino indistinta y paralelamente en varios lugares de manera independiente y posiblemente estén relacionados con los factores 
antrópicos, sumado a factores ecológicos y del comportamiento propio de la especie. Este mismo desplazamiento de A. cunicularia se está dando desde Brasil hacia la selva baja peruana (Gomes et al., 2013). Al respecto, Sick (1997) mencionó que A. cunicularia aprovecha los cambios en el paisaje forestal para ampliar su rango de distribución en colinas con muy poca vegetación en Río de Janeiro y en Brasilia. Los registros de A. cunicularia para la región central del Estado de Amazonas también sugieren que la especie está expandiendo su distribución a través de la Amazonia, luego de la amplia transformación de áreas forestales a pastos y tierras agrícolas (Aleixo \& Poletto, 2007). En el Parque Municipal do Sabiá, Uberlândia, Estado de Minas Gerais, un sitio de alta actividad humana, se han identificado lugares de selección y reutilización de sitios de nidificación de $A$. cunicularia (Martinelli, 2010). Adicionalmente, Cohn et al. (1997) efectuaron una evaluación ocho años después, en un área previamente inventariada, y encontraron 15 especies como nuevos colonizadores en el área; del mismo modo, Gomes et al. (2010) registraron la expansión de la distribución de Anodorhynchus hyacinthinus

Schistochlamys melanops en el Estado de Amazonas. Considerando estos antecedentes y el presente análisis, A. cunicularia podría estar adoptando la misma estrategia de ampliación de su área de distribución.

En el Perú es muy probable que las poblaciones de A. cunicularia hayan ingresado a la selva baja siguiendo la trayectoria de los valles interandinos que atraviesan la selva alta y confluyen, en la selva baja, con la mayoría de los ríos que componen la cuenca hidrográfica del río Amazonas. En las márgenes de los ríos se han establecido terrenos de cultivo, lo que ha traído como consecuencia que progresivamente la vegetación de árboles y arbustos de gran tamaño haya sido eliminada, para dar a paso a una mayor área cultivada. Esta situación podría haber generado condiciones propicias para que a lo largo de los años A. cunicularia progresivamente haya empezado a colonizar nuevos lugares en las partes bajas de la Amazonia.

Esta situación se ve reflejada en el caso de la selva baja sur oriental siguiendo el recorrido del rio Madre de Dios, que nace en las estribaciones orientales de la cordillera de Vilcanota (Cuzco), desciende por Pilcopata, donde se denomina río Alto Madre de Dios, continua su descenso por la llanura hasta la confluencia con el río Manú, donde es llamado río Madre de Dios. Haciendo el seguimiento de los reportes de numerosos observadores de aves, la especie es registrada durante todo el trayecto del rio Madre de Dios hasta llegar a selva baja por el Manu (TheCornellLab of Ornithology, 2020). La otra ruta es la postulada por Yábar (2008), que la especie está colonizando nuevas áreas desplazándose desde Bolivia hacia el Perú. En estas circunstancias, A. cunicularia ha ingresado a Perú desde el Acre, Brasil, donde existe una fuerte deforestación. Al respecto, Guilherme (2012) señala que algunas especies invasoras como Geranoaetus albicaudatus, Caracara plancus, Vanellus chilensis y A. cunicularia, se han ubicado principalmente en el extremo oriental del Estado, en la frontera con Perú, presumiblemente debido a la alta densidad de población humana y la existencia significativa de hábitats alterados. Esta parte de Acre está conectada al Estado de Rondônia por medio de una carretera que atraviesa muchos grandes ranchos ganaderos, lo que explica por qué algunas especies, como A. cunicularia, han colonizado el Estado, desde el Este, hacia Cruzeiro do Sul, así como áreas en el Sureste, en dirección a las ciudades de Brasiléia y Assis Brasil, en la frontera con Bolivia y Perú (Guilherme \& Santos, 2009; Guilherme \& Dantas, 2011).

\section{Conclusiones}

Los cambios ambientales que están ocurriendo en la Amazonia peruana estarían impactando en la diversidad de especies por dos caminos definidos: la destrucción del bosque primario por efecto de las actividades antropogénicas, que está ocasionando la perdida de diferentes especies de vertebrados e invertebrados; y por otra, la generación de nuevos hábitats, como los bosques secundarios en sus diversas caracterizaciones, que favorece la aparición de hábitats propicios para la colonización de especies provenientes de ambientes intervenidos.

Athene cunicularia, especie típica de las zonas áridas en las Vertientes Occidentales, la Puna y los altos Andes, desde hace veinte años está colonizando hábitats específicos producto de las etapas de sucesión como purmas y bosques secundarios, que se han generado como consecuencia de la devastación de los bosques primarios de la Amazonia; lo que sugiere que es una especie indicadora en lugares donde se produce la destrucción de los bosques y probablemente el avance del cambio climático.

Se propone que el avance de A. cunicularia en la colonización de la Amazonia, desde el lado peruano, sería siguiendo la ruta de los ríos que descienden por los valles interandinos, atraviesa la selva alta y llegan a la selva baja, acompañando el proceso de avance del establecimiento de terrenos de cultivo y asentamientos humanos.

\section{Agradecimientos}

El autor desea expresar su agradecimiento a la Escuela Profesional de Medicina Humana de la Universidad San Juan Bautista por el apoyo económico brindado para el desarrollo de la presente investigación.

De igual manera, al Dr. Marc Dourojeanni, profundo conocedor de la Amazonia peruana, por la exhaustiva y aguda revisión del manuscrito, así como por los aportes y recomendaciones efectuados. A don Manuel Plenge, Tarcisio Granizo, José Luis Venero, 
César Arana y Letty Salinas por la valiosa información proporcionada.

\section{Literatura citada}

Aleixo A. \& Poletto F. 2007. Birds of an open vegetation enclave in southern Brazilian Amazonia. The Wilson Journal of Ornithology, 119(4): 610-630. https://doi.org/10.1676/06-153.1.

Alonso J.Á., Metz M.R. \& Fine P.V.A. 2013. Habitat Specialization by Birds in Western Amazonian Whitesand Forests. Biotropica, 45(3): 365-372. DOI: $10.1111 /$ btp. 12020 .

BirdLife International. 2016. Athene cunicularia. The IUCN Red List of Threatened Species 2016: e.T22689353A93227732.

http://dx.doi.org/10.2305/IUCN.UK.2016-

3.RLTS.T22689353A93227732.en. Downloaded on 30 March 2020

Borsellino L. 2017. Nidificación exitosa de una pareja de lechucita vizcachera (Athene cunicularia) en una cueva artificial en la Ciudad Autónoma de Buenos Aires, Argentina. Notulas Faunísticas (Segunda Serie), 2017: 211. https://www.fundacionazara.org.ar/img/notulasfaunisticas/articulos/notula-211.pdf.

Butrón R. \& Tapia T. 2010. Aves. En: Figueroa J. \& Stucchi M. (eds). Biodiversidad de los alrededores de Puerto Maldonado. Línea base ambiental del EIA del lote 111, Madre de Dios, Perú. 149-179. Editorial: Ingeniería de proyectos y desarrollo. Lima, Perú. https://museohn.unmsm.edu.pe/docs/pub_ento/Figueroa $\% 20$ y\%20Stucchi,\%20ed\%202010\%20-

$\%$ 20Biodiversidad\%20de $\% 2010$ \% $\% 20$ alrededores $\% 20 \mathrm{de}$ $\% 20$ Puerto\%20Maldonado_2.pdf.

Canterbury G.E., Martin T.E., Petit D.R., Petit L.J. \& Bradford D.F. 2000. Bird communities and habitat as ecological indicators of forest condition in regional monitoring. Conservation Biology, 14(2): 544558. DOI: https://doi.org/10.1046/j.15231739.2000.98235.x.

Carrete M. \& Tella J.L. 2017. Behavioral Correlations Associated with Fear of Humans Differ between Rural and Urban Burrowing Owls. Front. Ecol. Evol., 5: 54. DOI: $10.3389 /$ fevo.2017.00054.

Cohn-Haft M., Whittaker A. \& Sttoufer P.C. 1997. A new look at the "species-poor" Central Amazon: the avifauna north of Manaus, Brazil. Ornithological Monographs, 48: 205-235. DOI: https://doi.org/10.2307/40157535.

Colwell R.K., Brehm G., Cardelús C., Gilman A. \& Longino J.T. 2008. Global Warming, Elevational Range Shifts, and Lowland Biotic Attrition in the Wet Tropics. Science, 322(5899): 258-261. DOI: 10.1126/science.1162547.

CRAP (Comité de Registros de Aves Peruanas). 2015. Reporte del Comité de Registros de Aves Peruanas (CRAP) del periodo 2014. Boletín UNOP (Unión de Ornitólogos del Perú), 10(2): 63-71. http://boletinunop.weebly.com/uploads/6/2/2/6/6226598 5/boletin_unop_vol._10_n\%C2\%BA2_2015_alta__crap.pdf.

De Sy V., Herold M., Achard F., Beuchle R., Clevers J.G.P.W., Lindquist E. \& Verchot L. 2015. Land use patterns and related carbon losses following deforestation in South America. Environmental Research Letters, 10(12): 124004. DOI: https://doi.org/10.1088/17489326/10/12/124004.
Delzo R., Zambrano D. \& Souza J. 2019. Segundo conteo de aves en los distritos de Callería, Manantay, Yarinacocha y zonas aledañas, Pucallpa. Revista Ciencia y Desarrollo. Universidad Alas Peruanas, 22(2): 7-30. http://dx.doi.org/10.21503/cyd.v22i2.1750.

Dourojeanni M.J. 2019a. Conservación de insectos en la amazonia. Ecología Aplicada, 18(2): 189-202. DOI: http://dx.doi.org/10.21704/rea.v18i2.1337.

Dourojeanni M.J. 2019b. Amazonia Peruana ¿Qué futuro? Pronaturaleza y Universidad Nacional Enrique Guzmán y Valle. Grijley Lima.

Fjeldså J. 2020. Rufous Twistwing (Cnipodectes superrufus). En: del Hoyo J., Elliott A., Sargatal J., Christie D.A. \& de Juana E. (eds.). Manual de las aves del mundo vivo. Lynx Edicions, Barcelona. Recuperado de https://www.hbw.com/node/204357 el 5 de abril de 2020.

Gaston K.J. 2005. Biodiversity and extinction: species and people. Progress in Physical Geography, 29(2): 239-247. https://doi.org/10.1191/0309133305pp445pr.

GBIF. 2020. Global Biodiversity Information Facility (GBIF). Consultado entre enero y mayo de 2020 de: https://www.gbif.org/.

Gomes F.B.R., Barreiros M.H.M. \& Fortes R. 2010. A distribuição do sanhaço-de-coleira Schistochlamys melanopis (Passeriformes: Thraupidae) e a influência de atividades humanas na ampliação de sua área de ocorrência. Atualidades Ornitológicas, 156: 4-5. https://coavap.files.wordpress.com/2012/07/gomes-f-br-m-h-m-barreiros-r-fortes-2010-a-distribuic3a7c3a3odo-sanhac3a7o-de-coleira-schistochlamys-melanopislatham-.

Gomes F.B.R., Barreiros M.H.M. \& Santana T.B.K. 2013. Novos registros da expansão geográfica de Athene cunicularia na Amazônia central com especial referência as atividades humanas. Atualidades Ornitológicas, $\quad 172$ : 12-14. https://coavap.files.wordpress.com/2012/07/gomes-etal_2013_athene-cunicularia.pdf.

Granizo T., Pacheco C., Ribadeneira M.B., Guerrero M. \& Suárez L. (Eds.). 2002. Libro Rojo de las Aves del Ecuador. Serie Libros Rojos del Ecuador, Tomo 2. SIMBIOE / Conservación Intemacional / EcoCiencia / Ministerio del Ambiente / UICN. Quito, Ecuador. https://biblio.flacsoandes.edu.ec/libros/digital/56484.pdf

Guilherme E. 2012. Birds of the Brazilian state of Acre: Diversity, zoogeography, and conservation. Revista Brasileira de Ornitologia, 20(4): 393-442. https://www.researchgate.net/publication/281872418_Bi rds_of_the_Brazilian_state_of_Acre_Diversity_zoogeog raphy_and_conservation.

Guilherme E. \& Santos M.P.D. 2009. Birds associated with bamboo forest in eastern Acre, Brasil. Bulletin of the British Ornithological Club, 129 (4): 229-240. https://www.researchgate.net/profile/Marcos_Santos26/p ublication/261147584_Birds_associated_with_bamboo_ forests_in_eastern_Acre_Brazil/links/0f31753357b8be2f f5000000.pdf.

Guilherme E. \& Dantas S.M. 2011. Avifauna of the upper Purus river, State of Acre, Brazil. Revista Brasileira de Ornitologia, $19 \quad$ (2): 185-199. https://www.researchgate.net/publication/286722763_A vifauna_of_the_Upper_Purus_River_State_of_Acre_Bra zil. 
Guilherme E., Marques E. L. \& Souza Santos G. 2018 Avifauna of a white-sand vegetation enclave in northwest Rondônia, Brazil: relevant records, body mass and morphometrics Bulletin of the British Ornithologists' Club, $\quad 138(4)$ : 286-306. https://doi.org/10.25226/bboc.v138i4.2018.a2

Harvey M.G., Seeholzer G.F., Cáceres D.A. , Winger B.M., Tello J.G., Hernández, F., Aponte M.A., Judy C.D., Figueroa S., Terrill R.S., Brown C.E., Alza L.A., Bravo G., Combe M., Custodio O., Quiñonez A., Urbay A., García W.A., Savit A.Z., Pezo F.W., Mauck III W.M. \& Barden O. 2014. The avian biogeography of an amazonian headwater: the upper Ucayali river, Peru. The Wilson Journal of Ornithology, 126(2): 179-191. DOI: https://doi.org/10.1676/13-135.1.

Lara M. 2020. Bioindicadores de la contaminación atmosférica. MoleQla: Revista de Ciencias de la Universidad Pablo de Olavide, (39): 30-34. https://www.upo.es/cms1/export/sites/upo/moleqla/docu mentos/Numero39/Numero-39.pdf.

Lepczyk C.A, Flather C.H., Radeloff V.C., Idgeon A.M., Hammer R.B. \& Liu J. 2008. Human Impacts on Regional Avian Diversity and Abundance. Conservation Biology, 22(2): $\quad 405-416 . \quad$ DOI: $10.1111 / \mathrm{j} .1523-$ 1739.2008.00881.x

Lincer J.L., Clark R.J., Fleming T.L. \& Sieradzki A. 2018. A Review of Burrowing Owl (Athene cunicularia) Literature Using Bibliometric Comparisons: Topical Bibliographies and Online Databases. Journal of Raptor Research, 52(2): 207-224. (1 June 2018). https://doi.org/10.3356/JRR-17-04.1.

Locatelli B., Kanninen M., Brockhaus M., Colfer C.J.P., Murdiyarso D. \& Santoso H. 2009. Ante un futuro incierto: cómo se pueden adaptar los bosques y las comunidades al cambio climático. Perspectivas forestales No. 5. CIFOR, Bogor, Indonesia. https://www.researchgate.net/publication/255710111_A nte_un_futuro_incierto_Como_se_pueden_adaptar_los_ bosques_y_las_comunidades_al_cambio_climatico\#full TextFileContent.

Macías-Duarte A. \& Conway C. J. 2015. Distributional Changes in the Western Burrowing Owl (Athene cunicularia hypugaea) in North America from 1967 to 2008," Journal of Raptor Research, 49(1): 75-83. https://doi.org/10.3356/JRR-14-00004.1.

Martinelli A.G. 2010. Observaciones sobre selección y reutilización de sitios de nidificación de la lechucita vizcachera Athene cunicularia (Strigiformes: Strigidae) en el Parque Municipal do Sabiá, Uberlândia, Estado de Minas Gerais, Brasil. Nótulas Faunísticas - Segunda Serie, $\quad 50$ : 1-6. https://www.academia.edu/1964826/Observaciones_sob re_selecci\%C3\%B3n_y_reutilizaci\%C3\%B3n_de_sitios _de_nidificaci\%C3\%B3n_de_Athene_cunicularia_Strigi formes_Strigidae_en_el_Parque_Municipal_do_Sabi\%C 3\%A1_Uber1\%C3\%A2ndia_Estado_de_Minas_Gerais_ Brasil.

Mamédio dos Santos D., Lima V., Burgos C., Vanderly M., Vitoria E.V. \& Nascimento de Oliveira K. 2017. Caracterização alimentar da Athene cunicularia (Strigiformes: Strigidae) (coruja buraqueira). Ciênc. anim. bras., Goiânia, 18: 1-9. https://www.revistas.ufg.br/vet/article/view/e-24506.
Medina C.A., W. Zelada, L. Pollack, Huamán E. \& Gómez A. 2013. Dieta de la lechuza de los arenales, Athene cunicularia, en Trujillo y en el Cerro Campana, La Libertad (Perú). Rebiol, 33(2): 99-106. https://revistas.unitru.edu.pe/index.php/facccbiol/article/ view/562.

MINAM. 2009. Mapa de Deforestación de la Amazonía Peruana 2000. Memoria Descriptiva. MINAM. Lima, Perú. http://sinia.minam.gob.pe/download/file/fid/39067.

MINAM. 2013. Diagnóstico para la Gestión de Cambio Climático en Ucayali. https://issuu.com/proyectopet1194/docs/ucayali_-_diagnostico_para_la_gesti.

MINAM. 2015. Cuantificación y Análisis de la Deforestación en la Amazonia Peruana en el período 2010-2011-20132014. MINAM. Lima, Perú. http://infobosques.com/portal/wpcontent/uploads/2017/0 3/Memoria_Descriptiva_Cambios_Cobertura_Bosque_2 014.pdf.

Miserendino R. 2007. Registro del chiñi (Athene cunicularia) para la Amazonia boliviana. Kempffiana, 3(2): 23-24. http://museonoelkempff.org/sitio/Informacion/KEMPFF IANA/Kempffiana\%203(2)/23-24.pd.

Mittermeier R.A., Mittermeier C.G., Brooks D.M., Pilgrim J.D., Konstant W.R. \& Fonseca G.A.B. 2003. Wilderness and biodiversity conservation. PNAS, 100(18): 1030910313. DOI: https://doi.org/10.1073/pnas.1732458100.

Nores M. 2000. Riqueza de especies en la fauna de aves amazónicas desde una perspectiva evolutiva. Emu Ornitología Austral, 100 (5): 419-430. DOI: 10.1071 / mu0007s.

Núñez M. 2008. Evaluación de comunidades de aves en bosques secundarios restaurados en potreros abandonados ubicados en la cuenca del Río Zapotal, Hojancha, Costa Rica. Tesis Mag. Sc. CATIE. Turrialba, CR.

http://repositorio.bibliotecaorton.catie.ac.cr/bitstream/ha ndle/11554/2041/Evaluacion_de_comunidades_de_aves. pdf? sequence $=1 \&$ is Allowed $=y$.

Plenge M.A. 2021. Species and subspecies of the birds of Peru / Especies y subespecies de las aves del Perú. Version last update: 14 January 2021. Unión de Ornitólogos del Perú. Consultado en setiembre de 2021 de: https://sites.google.com/site/boletinunop/subespecies.

Pulido V. \& Aguilar P. 1979. Artrópodos presentes en la dieta de la "lechuza de los arenales" en las Lomas de Lachay, Lima. Revista Peruana de Entomología, 22(1): 91-94.

Pulido V.M. 1982. La lechuza de los arenales de las lomas de Lachay, Lima-Perú. Zonas Áridas, 2: 75-78. DOI: http://dx.doi.org/10.21704/za.v2i1.1118.

Pulido V., Salinas L. \& Arana C. 2007. Aves en el desierto de Ica. La experiencia de Agrokasa. AGROKASA, Lima, Perú. Primera edición. http://fondoeditorial.unmsm.edu.pe/index.php/fondoedit orial/catalog/book/119.

Pulido V., Salinas L. \& Arana C. 2013. Aves del Desierto de la costa central del Perú. Edit. Barrón, Lima. http://fondoeditorial.unmsm.edu.pe/index.php/fondoedit orial/catalog/book/118

Pulido V., Salinas L., del Pino J. \& Arana C. 2021. Revisión del conocimiento actual y conservación de la lechuza de los arenales Athene cunicularia (Molina, 1782) en el Perú. Revista Peruana de Biología, 28(1): e19242. DOI: http://dx.doi.org/10.15381/rpb.v28i1.19242. 
Rebolo-Ifrán N., Tella J.L. \& Carrete M. 2017. Urban conservation hotspots: predation release allows the grassland-specialist burrowing owl to perform better in the city. Scientific Reports, 7: 3527. https://doi.org/10.1038/s41598-017-03853-z.

Richard E., Contreras D. \& Angeoletto F. 2017. Nuevo Registro del Chiñi (Athene cunicularia) en la Amazonía de Bolivia. Spizaetus, (23): 2-5. Boletín de la Red de Rapaces Neotropicales. https://assets.peregrinefund.org/docs/newsletters/spizaet us-23-spanish-2017-06-23_151619.pdf.

Rocha A.D., Branco J.O. \& Barrilli G.H.C. 2021. Prey ecology of the burrowing owl Athene cunicularia cunicularia (Molina, 1782) on the northern coast of Santa Catarina, Brazil. Studies on Neotropical Fauna and Environment. https://doi.org/10.1080/01650521.2020.1867953.

Rojas Briceño N.B., Barboza Castillo E., Maicelo Quintana J.L., Oliva Cruz S.M. \& Salas López R. 2019. Deforestación en la Amazonía peruana: Índices de cambios de cobertura y uso del suelo basado en SIG. Boletín de la Asociación de Geógrafos Españoles, (81): 2538. http://dx.doi.org/10.21138/bage.2538a.

Salas Á.D. \& Mancera N.J. 2020. Aves como indicadoras ecológicas de etapas sucesionales en un bosque secundario, Antioquia, Colombia. Revista de Biología Tropical, 68(1): 39. https://dx.doi.org/10.15517/rbt.v68i1.34913

Salinas L., Arana C. \& Pulido V. 2006. Diversidad, abundancia y conservación de aves en un agroecosistema del desierto de Ica, Perú. Revista Peruana de Biología, 13(3): 155-167. https://doi.org/10.15381/rpb.v13i3.2333.

Schulenberg T.S., Stotz D.F. \& Rico L. 2006. Mapas de distribución de las aves del Perú, versión 1.0. Medio ambiente, cultura y conservación (ECCo), The Field Museum. http://fm2.fieldmuseum.org/uw_test/birdsofperu.

Schulenberg T.S., Stotz, D.F., Lane D., O’Neill J. \& Parker T. 2010. Birds of Peru, revised and updated. Princeton University Press. Princeton, NJ.
Sekercioglu C.H., Schneider S.H., Fay J.P. \& Loarie S.R. 2008. Climate change, elevational range shifts, and bird extinctions. Conservation Biology, 22: 140-150. DOI: $10.1111 / \mathrm{j} .1523-1739.2007 .00852 . x$.

Sekercioglu Ç., Wormworth J. \& Primack R. 2011. The effects of climate change on tropical birds. Biological Conservation,

148(1):1-18. DOI: 10.1016/j.biocon.2011.10.019.

SERFOR. 2018. Libro Rojo de la Fauna Silvestre Amenazada del Perú. Primera edición. Serfor (Servicio Nacional Forestal y de Fauna Silvestre), Lima, Perú.

Sick H. 1997. Ornitología Brasileira. 2da Ed. Editora Nova Fronteira. Rio de Janeiro.

Stouffer P.C. 2020. Birds in fragmented Amazonian rainforest: Lessons from 40 years at the Biological Dynamics of Forest Fragments Project. The Condor, 122(3): 1-15. DOI: 10.1093/condor/duaa005.

TheCornellLab of Ornithology. 2020. Lechuza Terrestre (Athene cunicularia). En: eBird: proyecto del Laboratorio de Ornitología de Cornell. University of Cornell. Consultado entre enero y mayo de 2020 de: https://ebird.org/map/burowl?neg=true\&env.minX=.

Wade J. \& Belthoff J. 2016. Responses of female burrowing owls to alterations in clutch size: are burrowing owls determinate or indeterminate egg-layers? J. Raptor Res., 50(1): 84-91. DOI: 10.3356/rapt-50-01-84-91.1.

WWF. 2004. Monitoreo ecológico del manejo forestal en el trópico húmedo: una guía para operadores forestales y certificadores con énfasis en Bosques de Alto Valor para la Conservación. WWF Centroamérica. San José, CR. https://wwfeu.awsassets.panda.org/downloads/wwfca_m onitoreo.pdf.

Yábar V. 2008. First record of Glittering-bellied Emerald Chlorostilbon aureoventris for Peru. Cotinga, 30: 57-58. http://www.neotropicalbirdclub.org/wpcontent/uploads/2017/08/C30-Yabar.pd.

Zimmer K.J., Parker T.A., Isler M.L. \& Isler P.R. 1997. Survey of a southern Amazonian avifauna: the Alta Floresta region, Mato Grosso, Brazil. Ornithological Monographs, 48: 887-918. DOI: 10.2307/40157573. 
Tabla 1. Registro de observaciones de Athene cunicularia de la sierra y Vertiente Oriental de los Andes Peruanos.

\begin{tabular}{|c|c|c|}
\hline \multicolumn{2}{|c|}{ Región/Lugar de referencia } & \multirow{2}{*}{$\begin{array}{l}\text { Observador y año } \\
\text { Venero } 2020 .\end{array}$} \\
\hline Cusco: & La Convención & \\
\hline & Urubamba & Walker 2002, 2005, 2015; Venero 2015. \\
\hline & Acjanaco & Drucker 2013. \\
\hline & Sabaluyoc, Hacienda Meli & Tupayachi 2018. \\
\hline Ayacucho: & Lucanas & Venero \& Brokaw 1980. \\
\hline Puno: & Melgar & Venero 2012; Huamán-Tapara 2018. \\
\hline \multirow[t]{2}{*}{ Apurímac: } & Antabamba & Venero 2011; Baiker 2011. \\
\hline & Aymaraes & Venero 2011; Baiker 2011. \\
\hline \multirow[t]{8}{*}{ Madre de Dios: } & Collpa Mascoitania & $\begin{array}{l}\text { Maggini 2017; Bittermann 2017; Padilla 2018; Valdez 2018; } \\
\text { Losh 2018; Steele 2018; Svingen 2018; Barton 2018; García } \\
2019 .\end{array}$ \\
\hline & Manu & Yabar 2007. \\
\hline & Boca Manu Pueblo & Rainville 2015. \\
\hline & Manu, Pakitza & Vavrek 2018. \\
\hline & Amazon Manú Lodge & Oullette 2014; Vanier 2014. \\
\hline & Manu Birding Lodge & $\begin{array}{l}\text { Salas 2017; Jilahuanco 2017; Molina 2017; Maceda 2017; } \\
\text { García 2017; Gonzales 2017; Llactahuaman 2020. }\end{array}$ \\
\hline & Rio Madre de Dios, Isla Rolin & $\begin{array}{l}\text { Schulenberg 2012; Komar 2012; Andersen 2012; Johnson } \\
2012 .\end{array}$ \\
\hline & Manu Wildlife Center & $\begin{array}{l}\text { Ahlman 2005; Hill 2008; Neumuth 2017; } \\
\text { Whitebread 2017; Waters 2017; Whitebread } 2017 .\end{array}$ \\
\hline \multirow[t]{3}{*}{ Ucayali: } & Atalaya & Harvey 2014; Johnson 2015. \\
\hline & Coronel Portillo & Delzo et al., 2019; Pulido 2020 \\
\hline & Cashibo Ecolodge & Sales 2019; Rodríguez 2019; Bejarano 2019; Escajadillo 2019. \\
\hline \multirow[t]{6}{*}{ Loreto: } & Maynas & Olmos 2012; Cubas 2012; Fong 2018. \\
\hline & Amazonian River Islands & Bates 2018. \\
\hline & Explorama Lodge & Reina 2020; Morgan 2020; Beil Morgan 2020. \\
\hline & Islas del Rio Amazonas & Carnes 2011; Olmos 2012; Armas 2018. \\
\hline & Isla Joven Capironal & Arévalo 2020. \\
\hline & Yurimaguas & Mercurio 2016. \\
\hline Pasco: & Oxapampa, Pozuzo & Galván 2019, 2020. \\
\hline Junín: & Satipo & Chumpitaz 2018. \\
\hline \multirow[t]{2}{*}{ San Martín: } & Bellavista & Lane 2016; Johnson, 2016. \\
\hline & Tarapoto & Gonzales 2017. \\
\hline \multirow[t]{2}{*}{ Amazonas: } & Chachapoyas & September 2013. \\
\hline & Bagua Grande & Beadle 2008; Gonzales 2017, 2018. \\
\hline Huánuco: & Tingo María & Pinedo 2019. \\
\hline \multirow[t]{2}{*}{ Cajamarca: } & Cumbemayo & Bormann 2017. \\
\hline & Santa Rosa & Rodríguez 2019; Lozano 2019; Vera 2019. \\
\hline
\end{tabular}

Fuentes: TheCornellLab of Ornithology (2020); Schulenberg, et al. (2006); Harvey et al. (2014); Delzo et al. (2019). 


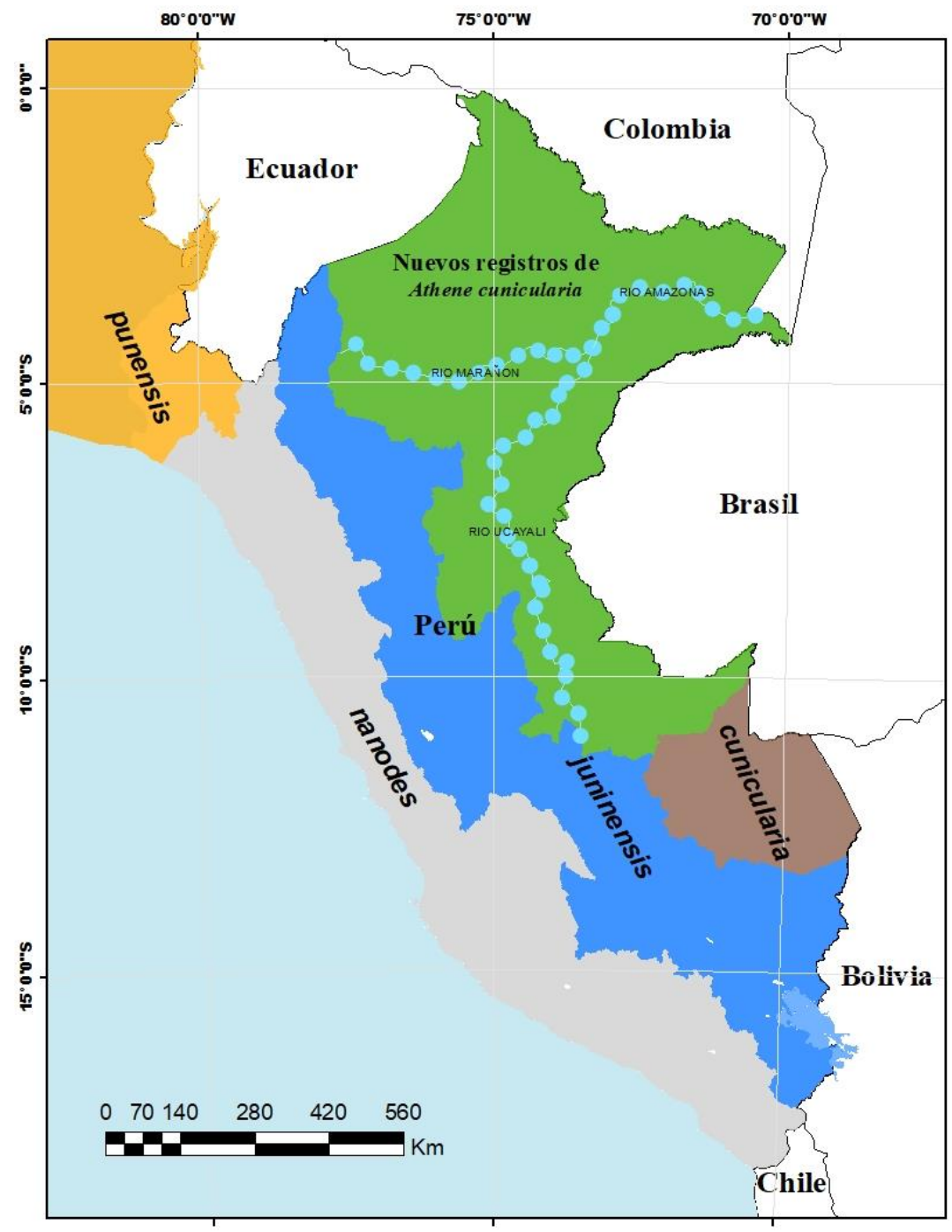

Fuentes: TheCornellLab of Ornithology (2020); Schulenberg, et al. (2006); Harvey et al. (2014); Delzo et al. (2019). y Pulido et al., 2021.

Figura 1. Distribución de subespecies y nuevos lugares de registros de Athene cunicularia en la Amazonia.

\footnotetext{
${ }^{1}$ Universidad Privada San Juan Bautista \Escuela Profesional de Medicina Humana. Av. José Antonio Lavalle N ${ }^{\circ}$ 302-304 (Ex Hacienda Villa), Chorrillos / Lima / Perú. victor.pulido@upsjb.edu.pe.
} 